\title{
Analytical Solution of Space-Time Fractional Fokker-Planck Equation by Homotopy Perturbation Sumudu Transform Method
}

\author{
Ravi Shanker Dubey, ${ }^{1}$ Badr Saad T. Alkahtani, ${ }^{2}$ and Abdon Atangana ${ }^{3}$ \\ ${ }^{1}$ Department of Mathematics, Yagyavalkya Institute of Technology, Jaipur 302022, India \\ ${ }^{2}$ Mathematics Department, College of Science, King Saud University, P.O. Box 1142, Riyadh 11989, Saudi Arabia \\ ${ }^{3}$ Institute for Groundwater Studies, Faculty of Natural and Agricultural Sciences, University of the Free State, \\ Bloemfontein 9300, South Africa \\ Correspondence should be addressed to Ravi Shanker Dubey; ravimath13@gmail.com
}

Received 27 May 2014; Revised 11 September 2014; Accepted 21 September 2014

Academic Editor: Samir B. Belhaouari

Copyright (C) 2015 Ravi Shanker Dubey et al. This is an open access article distributed under the Creative Commons Attribution License, which permits unrestricted use, distribution, and reproduction in any medium, provided the original work is properly cited.

\begin{abstract}
An efficient approach based on homotopy perturbation method by using Sumudu transform is proposed to solve some linear and nonlinear space-time fractional Fokker-Planck equations (FPEs) in closed form. The space and time fractional derivatives are considered in Caputo sense. The homotopy perturbation Sumudu transform method (HPSTM) is a combined form of Sumudu transform, homotopy perturbation method, and He's polynomials. The nonlinear terms can be easily handled by the use of He's polynomials. Some examples show that the HPSTM is an effective tool for solving many space time fractional partial differential equations.
\end{abstract}

\section{Introduction}

Fokker-Planck equation (FPE) was introduced by Adriaan Fokker and Max Planck to describe the time evolution of the probability density function of position and velocity of a particle, which is one of the classical widely used equations of statistical physics [1]. FPE arises in a number of different fields in natural sciences; Brownian motion [2] and the diffusion model of chemical reactions [3] are now largely employed, in various generalized forms, in physics, chemistry, engineering, and biology [1]. The FPE arises in kinetic theory [4] where it describes the evolution of the one-particle distribution function of a dilute gas with long-range collisions, such as a Coulomb gas. Some applications of this type of equations can be worked out in the works of $\mathrm{He}$ and $\mathrm{Wu}$ [5], Jumarie [6], Kamitani and Matsuba [7], Xu et al. [8], and Zak [9].

The general FPE for the motion of a concentration field $v(x, t)$ of one space variable $x$ at time $t$ has the form [1]

$$
\frac{\partial v(x, t)}{\partial t}=\left[-\frac{\partial A(x)}{\partial x}+\frac{\partial^{2} B(x)}{\partial x^{2}}\right] v(x, t)
$$

with initial condition

$$
v(0, x)=f(x), \quad x \in \mathscr{R},
$$

where $A(x)$ and $B(x)>0$ are called the drift and diffusion coefficients. This equation is also called the forward Kolmogorov equation. The drift and diffusion coefficients may also depend on time as

$$
\frac{\partial v(x, t)}{\partial t}=\left[-\frac{\partial A(x, t)}{\partial x}+\frac{\partial^{2} B(x, t)}{\partial x^{2}}\right] v(x, t) .
$$

There is a more general form of Fokker-Planck equation which is called the nonlinear Fokker-Planck equation. The nonlinear Fokker-Planck equation has important applications in various areas such as plasma physics, surface physics, population dynamics, biophysics, engineering, neurosciences, nonlinear hydrodynamics, polymer physics, laser physics, pattern formation, psychology, and so forth [10]. In the one variable case, the nonlinear FPE can be written as

$$
\frac{\partial v(x, t)}{\partial t}=\left[-\frac{\partial A(x, t, v)}{\partial x}+\frac{\partial^{2} B(x, t, v)}{\partial x^{2}}\right] v(x, t) .
$$


Due to vast range of applications of the FPE, a lot of work has been done to find numerical solution with this equation. In this context, the works of Buet et al. [11], Harrison [12], Palleschi et al. [13], Vanaja [14], and Zorzano et al. [15] are worth mentioning.

For generalization of classical integer order of FPE (4), the following equation can be introduced. The Fokker-Planck equation with fractional space derivative is a particular case of anomalous diffusion and Lévy flights (see[16-19]). This equation is called nonlinear FPE with space-time fractional derivatives [20]:

$$
\frac{\partial^{\alpha} v(x, t)}{\partial t^{\alpha}}=\left[-\frac{\partial A(x, t, v)}{\partial x}+\frac{\partial^{2} B(x, t, v)}{\partial x^{2}}\right] v(x, t)
$$

where $t>0, x>0$, and $0<\alpha \leq 1$. It can be obtained from the general Fokker-Planck equation by replacing the space and time derivatives by fractional derivatives operator. The function $v(x, t)$ is assumed to be a causal function of time and space. Particularly, for $\alpha=1$, the fractional FPE (5) reduces to the classical nonlinear FPE given by (4) in the case $x>0$.

In recent years, researchers have studied the fractional partial differential equations and the fractional FPE to investigate various scientific models [21-24]. In the present paper we obtain closed form solutions of a linear-nonlinear time fractional FPE using homotopy perturbation Sumudu transform method (HPSTM); see [25].

\section{Some Mathematical Preliminaries and Definitions}

2.1. Fundamental Properties of Fractional Calculus. Firstly, we mention some of the fundamental properties of the fractional calculus, that is, fractional derivatives and integrals.

The Riemann-Liouville fractional integral operator of order $\alpha>0$, of a function $f(t) \in C_{\mu}, \mu \geq-1$, is defined by implementing the integration operator $J^{\alpha}$ in the following manner:

$$
\begin{gathered}
J^{\alpha}[f(t)]=\frac{1}{\Gamma(\alpha)} \int_{0}^{t}(t-\tau)^{\alpha-1} f(\tau) d \tau, \quad \alpha>0, \\
J^{0}[f(t)]=f(t), \\
J^{\alpha} J^{\beta}[f(t)]=J^{\alpha+\beta} f(t), \\
J^{\beta} J^{\alpha}[f(t)]=J^{\alpha} J^{\beta} f(t), \\
J^{\alpha}\left(t^{\gamma}\right)=\frac{\Gamma(\gamma+1)}{\Gamma(\alpha+\gamma+1)} t^{\alpha+\gamma} .
\end{gathered}
$$

The fundamental properties of fractional integration and fractional differentiation have been introduced to the literature by Podlubny [26].
The fractional derivative of $f(t)$ in the Caputo sense is defined by the following relation [27]:

$$
\begin{aligned}
D_{t}^{\alpha} f(t) & =J^{m-\alpha} D^{n} f(t) \\
& =\frac{1}{\Gamma(n-\alpha)} \int_{0}^{t}(t-\tau)^{-\alpha+m-1} f^{(m)}(\tau) d \tau, \\
& m-1<\alpha \leq m,
\end{aligned}
$$

where $m \in N$ and $t>0$.

The relation between Riemann-Liouville fractional integral operator and the Caputo fractional derivative operator is

$$
\begin{gathered}
J_{t}^{\alpha} D_{t}^{\alpha} f(t)=f(t)-\sum_{k=0}^{m-1} \frac{f^{k}(0+) t^{k}}{k !}, \\
D^{-\alpha}[f(t)]=\frac{1}{\Gamma(\alpha)} \int_{0}^{t}(t-\tau)^{\alpha-1} f(\tau) d \tau, \\
0<\alpha \leq 1 .
\end{gathered}
$$

Further properties and applications can be found in $[26,28-$ 31].

2.2. The Sumudu Transform. In early 90s, Watugala [32] introduced a new integral transform named the Sumudu transform and applied it to the solution of ordinary differential equation in control engineering problems. The Sumudu transform is defined over the set of functions

$$
\begin{gathered}
A=\left\{f(t)\left|\exists M, \tau_{1}, \tau_{2}>0,\right| f(t) \mid<M e^{|t| \tau_{j}},\right. \\
\text { if } \left.t \in(-1)^{j} \times[0, \infty)\right\} ;
\end{gathered}
$$

the Sumudu transform is defined by

$$
\widetilde{G}(u)=S[f(t)]=\int_{0}^{\infty} f(u t) e^{-t} d t, \quad u \in\left(-\tau_{1}, \tau_{2}\right) .
$$

Sumudu transform has many useful and important properties like linear property, scale properties, shifting properties, duality with Laplace transforms, and so forth. Further detail and properties about this transform can be found in $[25,33-$ 37].

By using the Sumudu transform of multiple differentiation, we obtain

$$
\begin{aligned}
S\left[D_{t}^{\alpha} f(t)\right] & =u^{m-\alpha}\left[\frac{\widetilde{G}(u)}{u^{m}}-\sum_{k=0}^{m-1} \frac{f^{k}(0)}{u^{m-k}}\right] \\
& =\left[\frac{\widetilde{G}(u)}{u^{\alpha}}-\sum_{k=0}^{m-1} \frac{f^{k}(0+)}{u^{\alpha-k}}\right], \quad(m-1<\alpha \leq m),
\end{aligned}
$$

where $\widetilde{G}(u)=S[f(t)]$. 
2.3. The Mittag-Leffler Function. The Mittag-Leffler function which is a generalization of exponential function (see [38]) is as follows:

$$
E_{\alpha}(z)=\sum_{n=o}^{\infty} \frac{z_{n}}{\Gamma(\alpha n+1)} \quad(\alpha \in \mathscr{C}, \mathscr{R}(\alpha)>0) .
$$

2.4. Adomian Decomposition Method. The Adomian decomposition method (ADM) is a creative and effective method for exactly solving functional equations of various kinds. The method was developed by Adomian [39]. It is important to note that a large amount of research work has been devoted to the application of the ADM in a wide class of linear, nonlinear, ordinary, or partial differential equations. The decomposition method provides the solution as an infinite series in which each term can be determined easily.

\section{Solution of Fractional Differential Equations by Homotopy Perturbation Sumudu Transform Method (HPSTM)}

The homotopy perturbation method (HPM), introduced by He (see [40-43]), is a series expansion method used in the solution of nonlinear partial differential equations. The HPM uses a so-called convergence-control parameter to guarantee the convergence of approximation series over a given interval of physical parameters.

We illustrate the basic idea of this method by considering a general fractional nonlinear nonhomogeneous partial differential equation

$$
D_{t}^{\alpha} U(x, t)=L U(x, t)+N U(x, t)+f(x, t), \quad \alpha>0,
$$

with initial conditions

$$
\begin{gathered}
D_{0}^{k} U(x, 0)=g_{k}, \quad(k=0,1, \ldots, n-1), \\
D_{0}^{n} U(x, 0)=0, \quad n=[\alpha],
\end{gathered}
$$

where $D_{t}^{\alpha} U(x, t)$ is the Caputo fractional derivative of the function $U(x, t), L$ is the linear differential operator, $N$ represents the general nonlinear differential operator, and $f(x, t)$ is a known function.

Applying the Sumudu transform on both sides of (13), we get

$$
S\left[D_{t}^{\alpha} U(x, t)\right]=S[L U(x, t)]+S[N U(x, t)]+S[f(x, t)] .
$$

Using the property of the Sumudu transform, we have

$$
S[U(x, t)]=u^{\alpha} S[L U(x, t)+N U(x, t)]+g(x, t) .
$$

Operating with the inverse Sumudu transform on both sides of (16), we get

$$
U(x, t)=G(x, t)+S^{-1}\left[u^{\alpha} S[L U(x, t)+N U(x, t)]\right],
$$

where $G(x, t)$ represents the term arising from the function and the prescribed initial conditions.
Now we use the homotopy perturbation method

$$
U(x, t)=\sum_{n=0}^{\infty} p^{n} U_{n}(x, t)
$$

and the nonlinear term can be decomposed as

$$
N U(x, t)=\sum_{n=0}^{\infty} p^{n} H_{n}(U)
$$

on using He's polynomials $H_{n}(U)$ (see $[10,44]$ ) which are given by

$$
H_{n}\left(U_{0}, U_{1}, \ldots, U_{n}\right)=\frac{1}{n !} \frac{\partial^{n}}{\partial p^{n}}\left[N\left(\sum_{i=0}^{\infty} p^{i} U_{i}\right)\right]_{p=0} .
$$

Substituting (18) and (19) in (17), we get

$$
\begin{aligned}
& \sum_{n=0}^{\infty} p^{n} U_{n}(x, t) \\
& =G(x, t) \\
& \quad+p\left(S^{-1}\left[u^{\alpha} S\left[L \sum_{n=0}^{\infty} p^{n} U_{n}(x, t)+\sum_{n=0}^{\infty} p^{n} H_{n}(U)\right]\right]\right)
\end{aligned}
$$

which shows the coupling of the Sumudu transform and the HPM by using He's polynomials.

On collecting the coefficients of powers of $p$, we obtain

$$
\begin{aligned}
& p^{0}: U_{0}(x, t)=G(x, t) \\
& p^{1}: U_{1}(x, t)=S^{-1}\left[u^{\alpha} S\left\{L U_{0}(x, t)+H_{0}(U)\right\}\right] \\
& p^{2}: U_{2}(x, t)=S^{-1}\left[u^{\alpha} S\left\{L U_{1}(x, t)+H_{1}(U)\right\}\right]
\end{aligned}
$$

and similarly

$$
p^{n}: U_{n}(x, t)=S^{-1}\left[u^{\alpha} S\left\{L U_{n-1}(x, t)+H_{n-1}(U)\right\}\right] .
$$

Finally, we approximate the analytical solution $U(x, t)$, by truncated series

$$
U(x, t)=\lim _{N \rightarrow \infty} \sum_{n=0}^{N} U_{n}(x, t) .
$$

However, as we have seen in many cases, the exact solution in a closed form may be obtained. In addition, the decomposition series solutions generally converge very rapidly. Abbaoui and Cherruault [45] had defined the classical approach of convergence of this type of series.

\section{Solution of Fokker-Planck Fractional Differential Equation}

Solution of the following nonlinear space-time fractional Fokker-Planck equations (FPE) is

$$
\frac{\partial^{\alpha} v(x, t)}{\partial t^{\alpha}}=\left[-\frac{\partial A(x, t, v)}{\partial x}+\frac{\partial^{2} B(x, t, v)}{\partial x^{2}}\right] v(x, t)
$$


with the initial condition

$$
v(x, 0)=f(x) .
$$

Applying the Sumudu transform on both sides of (25), subject to initial condition (26), we have

$$
\begin{aligned}
S[v(x, t)]= & f(x)+u^{\alpha} S \\
& \times\left[-\frac{\partial A(x, t, v)}{\partial x} v(x, t)+\frac{\partial^{2} B(x, t, v)}{\partial x^{2}} v(x, t)\right],
\end{aligned}
$$

taking inverse Sumudu transform

$$
v(x, t)=f(x)+S^{-1}\left[u^{\alpha} S\left\{-\frac{\partial}{\partial x} \sum_{n=0}^{\infty} A_{n}+\frac{\partial^{2}}{\partial x^{2}} \sum_{n=0}^{\infty} B_{n}\right\}\right],
$$

where

$$
\begin{aligned}
& \sum_{n=0}^{\infty} A_{n}=A(x, t, v) \cdot v(x, t), \\
& \sum_{n=0}^{\infty} B_{n}=B(x, t, v) \cdot v(x, t) .
\end{aligned}
$$

Using HPM method, we get

$$
\begin{aligned}
& \sum_{n=0}^{\infty} p^{n} v_{n}(x, t) \\
& =f(x)+p \\
& \quad \times\left(S^{-1}\left[u^{\alpha} S\left\{-\sum_{n=0}^{\infty} p^{n} H_{n}(x, t, v)+\sum_{n=0}^{\infty} p^{n} H_{n}^{*}(x, t, v)\right\}\right]\right),
\end{aligned}
$$

where $H_{n}(x, t, v)$ and $H_{n}^{*}(x, t, v)$ are He's polynomials that represent the nonlinear terms. The He's polynomials are given by

$$
\begin{aligned}
& \sum_{n=0}^{\infty} H_{n}(x, t, v)=\frac{\partial}{\partial x}\left(\sum_{n=0}^{\infty} A_{n}\right), \\
& \sum_{n=0}^{\infty} H_{n}^{*}(x, t, v)=\frac{\partial^{2}}{\partial x^{2}}\left(\sum_{n=0}^{\infty} B_{n}\right) .
\end{aligned}
$$

Using the above equation we can collect the coefficient of power of $p$

$$
\begin{aligned}
& p^{0}: v_{0}(x, t)=f(x), \\
& p^{1}: v_{1}(x, t)=S^{-1}\left[u^{\alpha} S\left\{\left[-H_{0}+H_{0}^{*}\right]\right\}\right], \\
& p^{2}: v_{2}(x, t)=S^{-1}\left[u^{\alpha} S\left\{\left[-H_{1}+H_{1}^{*}\right]\right\}\right]
\end{aligned}
$$

and similarly

$$
p^{n}: v_{n}(x, t)=S^{-1}\left[u^{\alpha} S\left\{\left[-H_{n-1}+H_{n-1}^{*}\right]\right\}\right] .
$$

Finally, we approximate the analytical solution $v_{n}(x, t)$ by truncated series [25]

$$
v(x, t)=\lim _{N \rightarrow \infty} \sum_{n=0}^{N} v_{n}(x, t) ;
$$

the series solutions of the above equation converge very rapidly $[25,45]$.

\section{Numerical Examples}

In this section we will illustrate the HPSTM techniques by several examples. These examples are somewhat artificial in the sense that the exact answer, for the special case $\alpha=1$, is known in advance and the initial and boundary conditions are directly taken from this answer. Nonetheless, such an approach is needed to evaluate the accuracy of the analytical techniques and to examine the effect of varying the order of the space- and time-fractional derivatives on the behavior of the solution. All the results are calculated by using the symbolic calculus software MATLAB.

Example 1. Consider the nonlinear time fractional FPE

$$
D_{t}^{\alpha} v(x, t)=\left[-\frac{\partial}{\partial x}\left(3 v-\frac{x}{2}\right)+\frac{\partial^{2}}{\partial x^{2}}(x v)\right] v(x, t),
$$

where $t>0, x>0,0<\alpha \leq 1$, and $D_{t}^{\alpha}$ is the Caputo fractional derivative defined by (7) and initial condition is

$$
v(x, 0)=x .
$$

Then solution is given by $v(x, t)=x E_{\alpha}\left(t^{\alpha}\right)$.

Solution 1. On using the method defined in Section 4, we get the coefficient of powers of $p$

$$
\begin{aligned}
& p^{0}: v_{0}(x, t)=x, \\
& p^{1}: v_{1}(x, t)=\frac{x t^{\alpha}}{\Gamma(\alpha+1)}, \\
& p^{2}: v_{2}(x, t)=\frac{x t^{2 \alpha}}{\Gamma(2 \alpha+1)}, \\
& p^{3}: v_{3}(x, t)=\frac{x t^{3 \alpha}}{\Gamma(3 \alpha+1)},
\end{aligned}
$$

hence, $v(x, t)$ is

$$
\begin{gathered}
v(x, t)=x\left(1+\frac{t^{\alpha}}{\Gamma(\alpha+1)}+\frac{t^{2 \alpha}}{\Gamma(2 \alpha+1)}+\frac{t^{3 \alpha}}{\Gamma(3 \alpha+1)}+\cdots\right) \\
v(x, t)=x E_{\alpha}\left(t^{\alpha}\right) .
\end{gathered}
$$

Remark 2. Setting $\alpha=1$, Example 1 reduces to nonlinear FPE

$$
\frac{\partial}{\partial t} v(x, t)=\left[-\frac{\partial}{\partial x}\left(3 v-\frac{x}{2}\right)+\frac{\partial^{2}}{\partial x^{2}}(x v)\right] v(x, t)
$$


with initial condition

$$
v(x, 0)=x,
$$

and a solution as

$$
v(x, t)=x e^{t} .
$$

Example 3. Consider the nonlinear time fractional FPE

$$
\begin{aligned}
& D_{t}^{\alpha} v(x, t) \\
& \quad=\left[-\frac{\partial}{\partial x}\left(\frac{4 v}{x}-\frac{x}{3}\right)+\frac{\partial^{2}}{\partial x^{2}}(v)\right] v(x, t), \quad x>0, t>0,
\end{aligned}
$$

where $t>0, x>0,0<\alpha \leq 1$, and $D_{t}^{\alpha}$ is Caputo fractional derivative defined by (7) and initial condition is

$$
v(x, 0)=x^{2} .
$$

Then solution is given by $v(x, t)=x^{2} E_{\alpha}\left(t^{\alpha}\right)$.

Solution 2. By using the method defined in Section 4 , we get the coefficient of power of $p$

$$
\begin{aligned}
& p^{0}: v_{0}(x, t)=x^{2}, \\
& p^{1}: v_{1}(x, t)=\frac{x^{2} t^{\alpha}}{\Gamma(\alpha+1)}, \\
& p^{2}: v_{2}(x, t)=\frac{x^{2} t^{2 \alpha}}{\Gamma(2 \alpha+1)}, \\
& p^{3}: v_{3}(x, t)=\frac{x^{2} t^{3 \alpha}}{\Gamma(3 \alpha+1)} \cdots ;
\end{aligned}
$$

hence, $v(x, t)$ is

$$
\begin{gathered}
v(x, t) \\
=x^{2}\left(1+\frac{t^{\alpha}}{\Gamma(\alpha+1)}+\frac{t^{2 \alpha}}{\Gamma(2 \alpha+1)}+\frac{t^{3 \alpha}}{\Gamma(3 \alpha+1)}+\cdots\right) \\
v(x, t)=x^{2} E_{\alpha}\left(t^{\alpha}\right) .
\end{gathered}
$$

Remark 4. Setting $\alpha=1$, Example 3 reduces to nonlinear FPE

$$
\begin{aligned}
& \frac{\partial}{\partial t} v(x, t) \\
& \quad=\left[-\frac{\partial}{\partial x}\left(\frac{4 v}{x}-\frac{x}{3}\right)+\frac{\partial^{2}}{\partial x^{2}}(v)\right] v(x, t), \quad x>0, t>0
\end{aligned}
$$

with initial condition

$$
v(x, 0)=x^{2}
$$

and a solution as

$$
v(x, t)=x^{2} e^{t}
$$

Example 5. Consider the nonlinear time fractional FPE

$$
\begin{aligned}
& D_{t}^{\alpha} v(x, t) \\
& \quad=\left[-\frac{\partial}{\partial x}(x)+\frac{\partial^{2}}{\partial x^{2}}\left(\frac{x^{2}}{2}\right)\right] v(x, t), \quad x>0, t>0,
\end{aligned}
$$

where $t>0, x>0,0<\alpha \leq 1$, and $D_{t}^{\alpha}$ is Caputo fractional derivative defined by (7) and initial condition is

$$
v(x, 0)=x .
$$

Solution 3. By using the method defined in Section 4, we get the coefficient of $p$

$$
\begin{aligned}
& p^{0}: v_{0}(x, t)=x \\
& p^{1}: v_{1}(x, t)=\frac{x t^{\alpha}}{\Gamma(\alpha+1)}, \\
& p^{2}: v_{2}(x, t)=\frac{x t^{2 \alpha}}{\Gamma(2 \alpha+1)} \\
& p^{3}: v_{3}(x, t)=\frac{x t^{3 \alpha}}{\Gamma(3 \alpha+1)}
\end{aligned}
$$

hence, $v(x, t)$ is

$$
\begin{gathered}
v(x, t)=x\left(1+\frac{t^{\alpha}}{\Gamma(\alpha+1)}+\frac{t^{2 \alpha}}{\Gamma(2 \alpha+1)}+\frac{t^{3 \alpha}}{\Gamma(3 \alpha+1)}+\cdots\right) \\
v(x, t)=x E_{\alpha}\left(t^{\alpha}\right) .
\end{gathered}
$$

Remark 6. Setting $\alpha=1$ in problem, Example 5 reduces to nonlinear FPE

$$
\begin{array}{r}
\frac{\partial}{\partial t} v(x, t)=\left[-\frac{\partial}{\partial x}(x)+\frac{\partial^{2}}{\partial x^{2}}\left(\frac{x^{2}}{2}\right)\right] v(x, t), \\
x>0, \quad t>0,
\end{array}
$$

with initial condition

$$
v(x, 0)=x
$$

and a solution as

$$
v(x, t)=x e^{t}
$$

The approximate solutions for (42) and (49) shown in Tables 1 and 2, respectively, were obtained for different values of $x$ and $t$ by using the homotopy perturbation Sumudu transform method. The exact solutions of (42) and (49) are $v(x, t)=$ $x^{2} e^{t}$ and $v(x, t)=x e^{t}$, respectively. The accuracy of our approximate solutions can be improved by computing more terms of the approximate solutions. Both the methods viz. ADM and HPSTM are used adroitly to obtain the exact values and get comparisons. From the numerical results in Tables 1 and 2, it is easy to conclude that the solution continuously depends on the space-fractional derivative and the approximate solutions of (42) and (49) obtained by using 
TABLE 1: Comparison study for solution of (42) for $\alpha=1$, when $t=0.2,0.4,0.6$ and $x=0.25,0.50,0.75,1.0$. by various methods.

\begin{tabular}{lcccc}
\hline$t$ & $x$ & HPSTM & ADM (see [20]) & EXACT (see [20]) \\
\hline 0.2 & 0.25 & 0.076333 & 0.076333 & 0.076338 \\
0.2 & 0.50 & 0.305333 & 0.305333 & 0.305351 \\
0.2 & 0.75 & 0.687000 & 0.687000 & 0.687039 \\
0.2 & 1.00 & 1.221333 & 1.221333 & 1.221403 \\
0.4 & 0.25 & 0.093167 & 0.093167 & 0.093239 \\
0.4 & 0.50 & 0.372667 & 0.372667 & 0.372956 \\
0.4 & 0.75 & 0.838500 & 0.838500 & 0.839151 \\
0.4 & 1.00 & 1.490667 & 1.490667 & 1.491825 \\
0.6 & 0.25 & 0.113500 & 0.113500 & 0.113882 \\
0.6 & 0.50 & 0.454000 & 0.454000 & 0.455530 \\
0.6 & 0.75 & 1.021500 & 1.021500 & 1.024942 \\
0.6 & 1.00 & 1.816000 & 1.816000 & 1.822119 \\
\hline
\end{tabular}

TABLE 2: Comparison study for solution of (49) for $\alpha=1$, when $t=0.2,0.4,0.6$ and $x=0.25,0.50,0.75,1.0$. by various methods.

\begin{tabular}{lcccc}
\hline$t$ & $x$ & HPSTM & ADM (see [20]) & EXACT (see [20]) \\
\hline 0.2 & 0.25 & 0.305333 & 0.305333 & 0.305351 \\
0.2 & 0.50 & 0.610667 & 0.610667 & 0.610701 \\
0.2 & 0.75 & 0.916000 & 0.916000 & 0.916052 \\
0.2 & 1.00 & 1.221333 & 1.221333 & 1.221403 \\
0.4 & 0.25 & 0.372667 & 0.372667 & 0.372956 \\
0.4 & 0.50 & 0.745333 & 0.745333 & 0.745912 \\
0.4 & 0.75 & 1.118000 & 1.118000 & 1.118869 \\
0.4 & 1.00 & 1.490667 & 1.490667 & 1.491825 \\
0.6 & 0.25 & 0.454000 & 0.454000 & 0.455530 \\
0.6 & 0.50 & 0.908000 & 0.908000 & 0.911059 \\
0.6 & 0.75 & 1.362000 & 1.362000 & 1.366589 \\
0.6 & 1.00 & 1.816000 & 1.816000 & 1.822119 \\
\hline
\end{tabular}

the Adomian decomposition method are the same as those obtained by the homotopy perturbation Sumudu transform method. It is to be noted that only the fourth-order term of the series of the abovesaid methods was used in evaluating the approximate solutions for Tables 1 and 2 .

\section{Conclusion}

Within the scope of the HPSTM, we derive the solution of the nonlinear time fractional Fokker-Planck equations. We made use of the Caputo derivative because it allows usual initial conditions. The numerical result shows that the method used is very simple and straightforward to implement.

\section{Conflict of Interests}

The authors declare that there is no conflict of interests regarding the publication of this paper.

\section{Acknowledgment}

The authors are grateful to the anonymous referee for his/her careful reading and many corrections/suggestions to improve this paper. This project was supported by Deanship of Scientific Research, College of Science Research Center, King Saud University

\section{References}

[1] H. Risken, The FokkerPlanck Equation: Method of Solution and Applications, Springer, Berlin, Germany, 1989.

[2] S. Chandresekhar, "Stochastic problems in physics and astronomy," Reviews of Modern Physics, vol. 15, pp. 1-89, 1943.

[3] H. A. Kramers, "Brownian motion in a field of force and the diffusion model of chemical reactions," Physica, vol. 7, pp. 284304, 1940.

[4] A. Fokker, "The median energy of rotating electrical dipoles in radiation fields," Annalen Der Physik, vol. 43, pp. 810-820, 1914.

[5] J. H. He and X. H. Wu, "Construction of solitary solution and compaction-like solution by variational iteration method," Chaos Solitons \& Fractals, vol. 29, pp. 108-113, 2006.

[6] G. Jumarie, "Fractional Brownian motions via random walk in the complex plane and via fractional derivative, comparison and further results on their Fokker-Planck equations," Chaos Solitons \& Fractals, vol. 22, pp. 907-925, 2004.

[7] Y. Kamitani and I. Matsuba, "Self-similar characteristics of neural networks based on Fokker-Planck equation," Chaos Solitons \& Fractals, vol. 20, no. 2, pp. 329-335, 2004.

[8] Y. Xu, F. Y. Ren, J. R. Liang, and W. Y. Qiu, "Stretched Gaussian asymptotic behavior for fractional Fokker-Planck equation on fractal structure in external force fields," Chaos, Solitons \& Fractals, vol. 20, no. 3, pp. 581-586, 2004.

[9] M. Zak, "Expectation-based intelligent control," Chaos Solitons \& Fractals, vol. 28, pp. 616-626, 2006.

[10] S. T. Mohyud-Din, M. A. Noor, and K. I. Noor, "Traveling wave solutions of seventh-order generalized $\mathrm{KdV}$ equations using He's polynomials," International Journal of Nonlinear Sciences and Numerical Simulation, vol. 10, no. 2, pp. 227-233, 2009.

[11] C. Buet, S. Dellacherie, and R. Sentis, "Numerical solution of an ionic Fokker-Planck equation with electronic temperature," SIAM Journal on Numerical Analysis, vol. 39, no. 4, pp. 12191253, 2001.

[12] G. W. Harrison, "Numerical solution of the Fokker-Planck equation using moving finite elements," Numerical Methods for Partial Differential Equations, vol. 4, no. 3, pp. 219-232, 1988.

[13] V. Palleschi, F. Sarri, G. Marcozzi, and M. R. Torquati, "Numerical solution of the Fokker-Planck equation: a fast and accurate algorithm," Physics Letters A, vol. 146, no. 7-8, pp. 378-386, 1990.

[14] V. Vanaja, "Numerical solution of a simple Fokker-Planck equation," Applied Numerical Mathematics, vol. 9, no. 6, pp. 533540, 1992.

[15] M. P. Zorzano, H. Mais, and L. Vazquez, "Numerical solution of two-dimensional Fokker-Planck equations," Applied Mathematics and Computation, vol. 98, no. 2-3, pp. 109-117, 1999.

[16] A. A. Dubkov, B. Spagnolo, and V. V. Uchaikin, "Lévy flight superdiffusion: an introduction," International Journal of Bifurcation and Chaos in Applied Sciences and Engineering, vol. 18, no. 9, pp. 2649-2672, 2008. 
[17] A. A. Dubkov and B. Spagnolo, "Acceleration of diffusion in randomly switching potential with supersymmetry," Physical Review E, vol. 72, no. 4, part 1, Article ID 041104, 8 pages, 2005.

[18] A. Dubkov and B. Spagnolo, "Generalized Wiener process and Kolmogorov's equation for diffusion induced by non-Gaussian noise source," Fluctuation and Noise Letters, vol. 5, no. 2, pp. L267-L274, 2005.

[19] A. Dubkov and B. Spagnolo, "Langevin approach to Lévy flights in fixed potentials: exact results for stationary probability distributions," Acta Physica Polonica B, vol. 38, no. 5, pp. 17451758, 2007.

[20] Z. Odibat and S. Momani, "Numerical solution of FokkerPlanck equation with space- and time-fractional derivatives," Physics Letters A, vol. 369, pp. 349-358, 2007.

[21] Q. Yang, F. Liu, and I. Turner, "Computationally efficient numerical methods for time and space-fractional FokkerPlanck equations," Physica Scripta, vol. 2009, no. T136, Article ID 014026, 7 pages, 2009.

[22] Q. Yang, F. Liu, and I. Turner, "Stability and convergence of an effective numerical method for the time-space fractional Fokker-Planck equation with a nonlinear source term," International Journal of Differential Equations, vol. 2010, Article ID 464321, 22 pages, 2010.

[23] A. Yildirim, "Analytical approach to Fokker-Planck equation with space- and timefractional derivatives by means of the homotopy perturbation method," Journal of King Saud University (Science), vol. 22, pp. 257-264, 2010.

[24] P. Zhuang, F. Liu, V. Anh, and I. Turner, "Numerical treatment for the fractional Fokker-Planck equation," Anziam Journal, vol. 48, pp. C759-C774, 2007.

[25] A. Atangana and A. Kılıçman, "The use of Sumudu transform for solving certain nonlinear fractional heat-like equations," Abstract and Applied Analysis, vol. 2013, Article ID 737481, 12 pages, 2013.

[26] F. Mainardi, "Fractional calculus: some basic problems in continuum and statistical mechanics," in Fractals and Fractional Calculus in Continuum Mechanics, A. Carpinteri and F. Mainardi, Eds., vol. 378, pp. 291-348, Springer, New York, NY, USA, 1997.

[27] M. Caputo, Elasticitáe Dissipazione, Zanichelli, Bologna, Italy, 1969, [Italian].

[28] G. Jumarie, "Laplace's transform of fractional order via the Mittag-Leffler function and modified Riemann-Liouville derivative," Applied Mathematics Letters, vol. 22, no. 11, pp. 1659-1664, 2009.

[29] I. Podlubny, Fractional Differential Equations, vol. 198 of Mathematics in Science and Engineering, Academic Press, New York, NY, USA, 1999.

[30] F. Mainardi, "The fundamental solutions for the fractional diffusion-wave equation," Applied Mathematics Letters, vol. 9, no. 6, pp. 23-28, 1996.

[31] F. Mainardi, Y. Luchko, and G. Pagnini, "The fundamental solution of the space-time fractional diffusion equation," Fractional Calculus \& Applied Analysis, vol. 4, no. 2, pp. 153-192, 2001.

[32] G. K. Watugala, "Sumudu transform: a new integral transform to solve differential equations and control engineering problems," International Journal of Mathematical Education in Science and Technology, vol. 24, no. 1, pp. 35-43, 1993.

[33] F. B. M. Belgacem, "Applications of the sumudu transform to indefinite periodic parabolic equations," in Proceedings of the 6th International Conference on Mathematical Problems
\& Aerospace Sciences (ICNPAA '06), chapter 6, pp. 51-60, Cambridge Scientific, Cambridge, UK, 2007.

[34] F. B. Belgacem, A. A. Karaballi, and S. L. Kalla, "Analytical investigations of the Sumudu transform and applications to integral production equations," Mathematical Problems in Engineering, vol. 2003, no. 3, pp. 103-118, 2003.

[35] J.-H. He, "A coupling method of a homotopy technique and a perturbation technique for non-linear problems," International Journal of Non-Linear Mechanics, vol. 35, no. 1, pp. 37-43, 2000.

[36] V. B. L. Chaurasia, R. S. Dubey, and F. B. M. Belgacem, "Fractional radial diffusion equation analytical solution via Hankel and Sumudu transforms," Mathematics in Engineering, Science and Aerospace, vol. 3, no. 2, pp. 1-10, 2012.

[37] Q. D. Katatbeh and F. B. M. Belgacem, "Applications of the Sumudu transform to fractional differential equations," Nonlinear Studies, vol. 18, no. 1, pp. 99-112, 2011.

[38] G. M. Mittag-Leffler, Sur la Nouvelle Fonction Ea(x), vol. 137, C. R. Academy of Science, Paris, France, 1903.

[39] G. Adomian, Solving Frontier Problems of Physics: The Decomp Osition Method, Kluwer Academic, Boston, Mass, USA, 1994.

[40] J.-H. He, "The homotopy perturbation method nonlinear oscillators with discontinuities," Applied Mathematics and Computation, vol. 151, no. 1, pp. 287-292, 2004.

[41] J.-H. He, "Homotopy perturbation technique," Computer Methods in Applied Mechanics and Engineering, vol. 178, no. 3-4, pp. 257-262, 1999.

[42] A. A. Hemeda, "Homotopy perturbation method for solving systems of nonlinear coupled equations," Applied Mathematical Sciences, vol. 6, no. 93-96, pp. 4787-4800, 2012.

[43] J. Saberi-Nadjafi and A. Ghorbani, "He's homotopy perturbation method: an effective tool for solving nonlinear integral and integro-differential equations," Computers \& Mathematics with Applications, vol. 58, no. 11-12, pp. 2379-2390, 2009.

[44] A. Ghorbani, "Beyond Adomian polynomials: he polynomials," Chaos, Solitons and Fractals, vol. 39, no. 3, pp. 1486-1492, 2009.

[45] K. Abbaoui and Y. Cherruault, "New ideas for proving convergence of decomposition methods," Computers \& Mathematics with Applications, vol. 29, no. 7, pp. 103-108, 1995. 


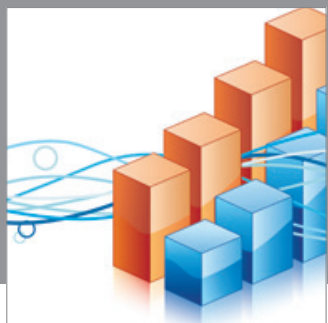

Advances in

Operations Research

mansans

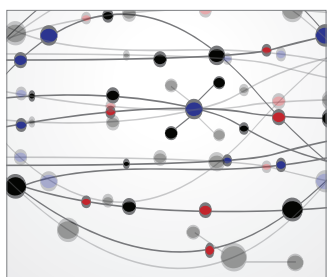

The Scientific World Journal
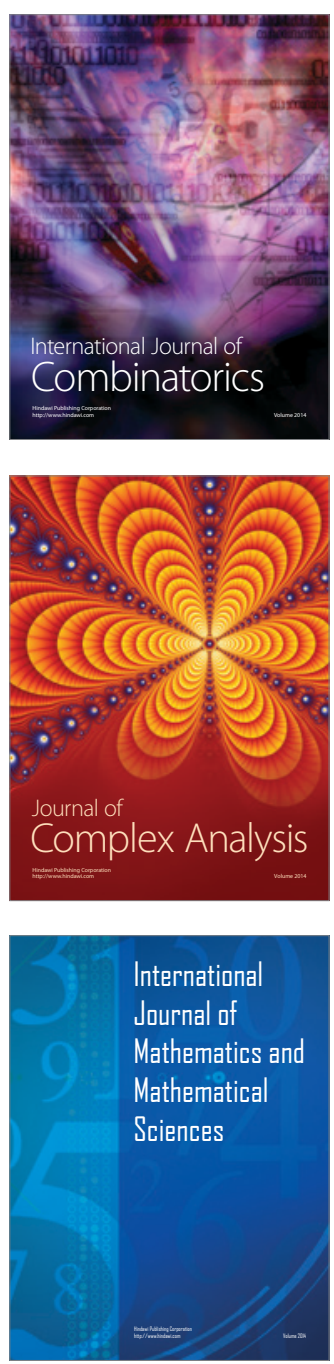
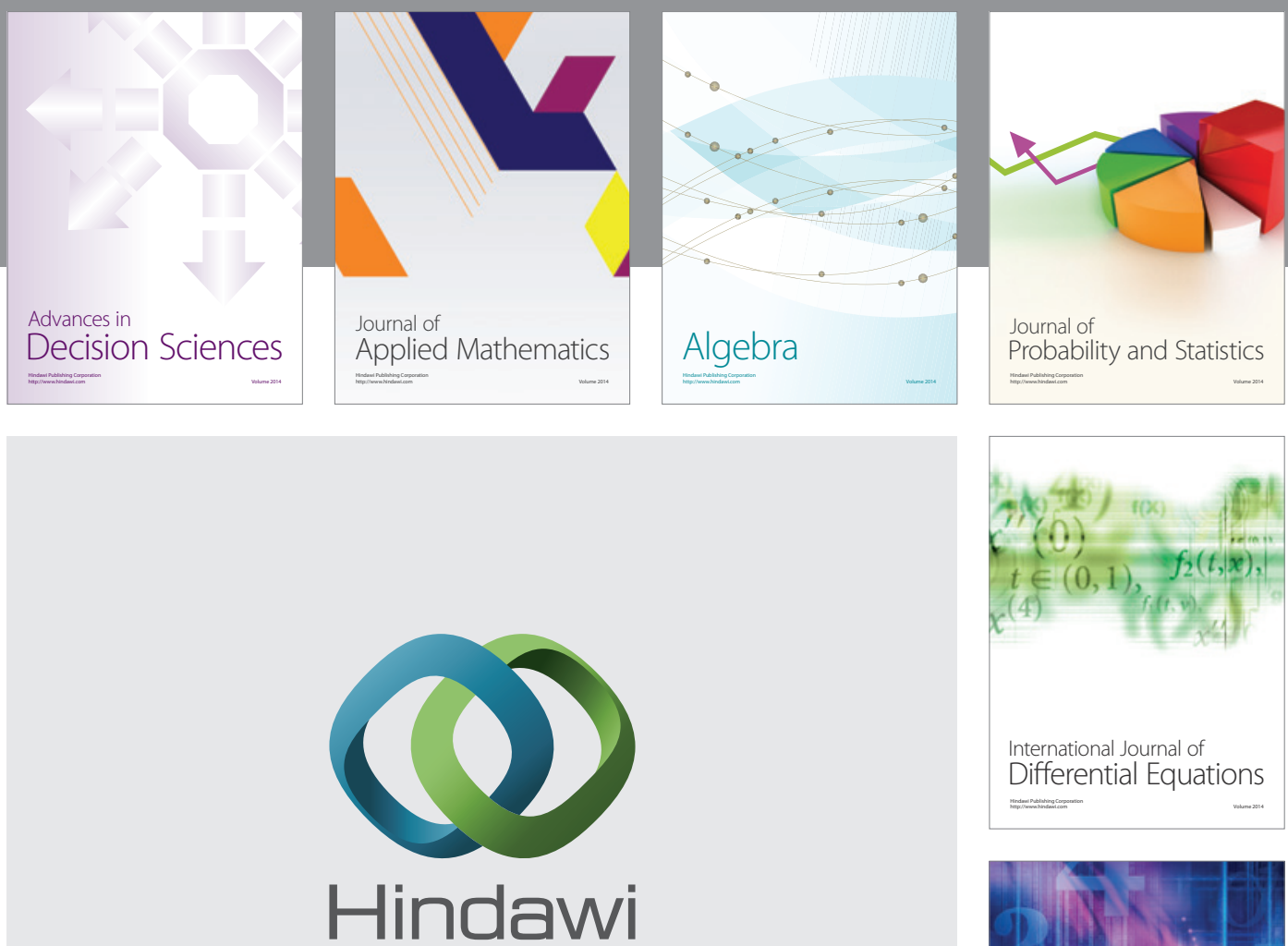

Submit your manuscripts at http://www.hindawi.com
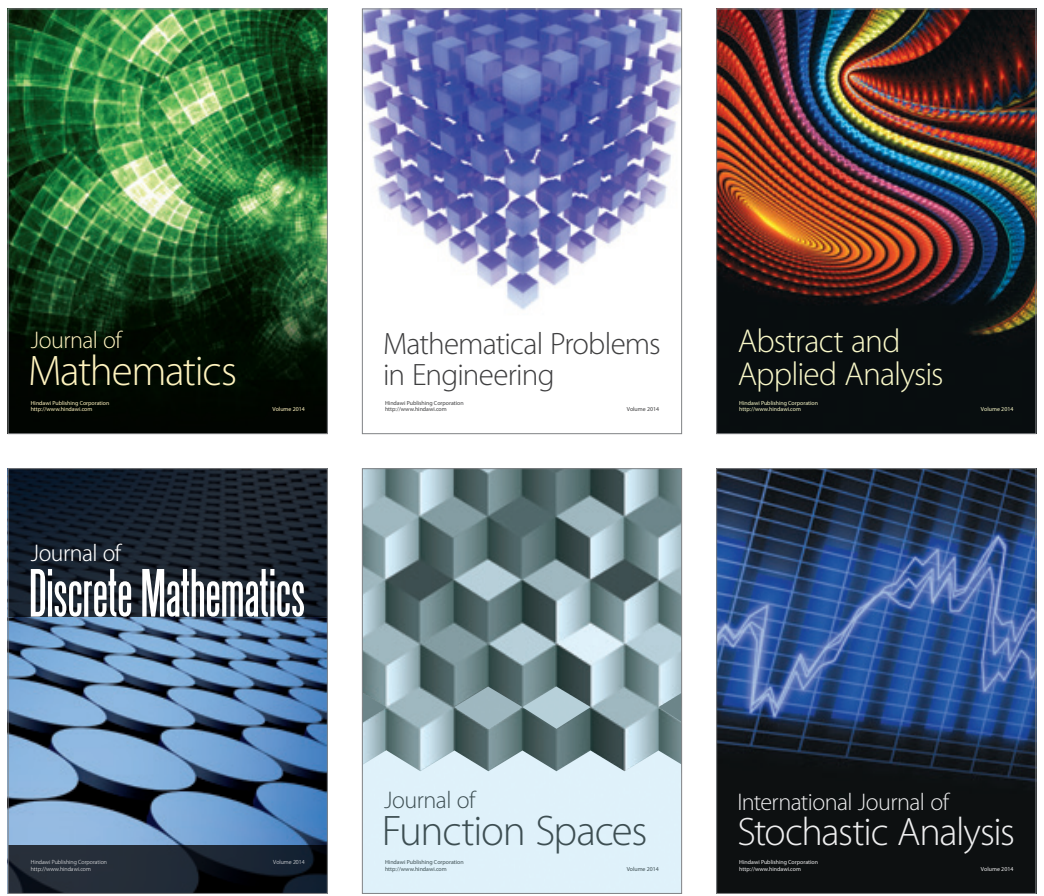

Journal of

Function Spaces

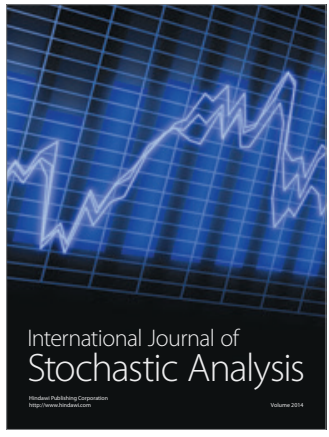

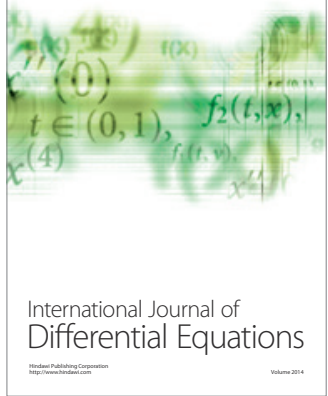
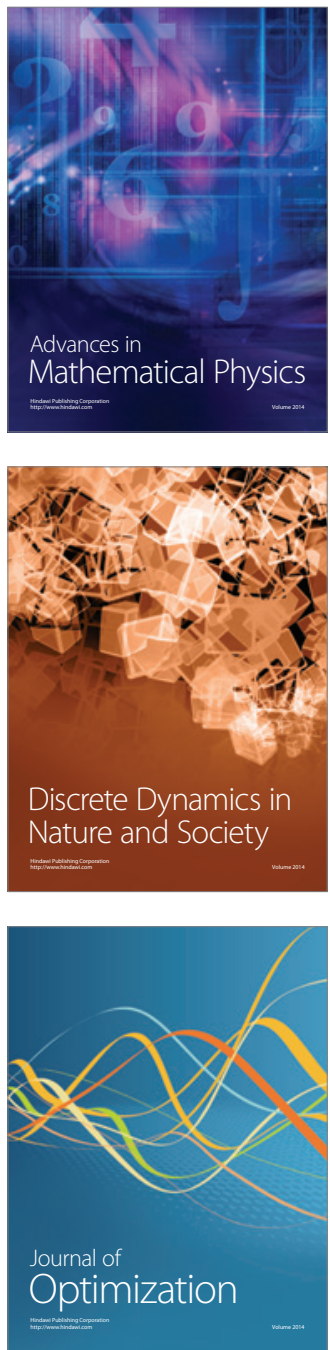